\title{
The Severity of Muscle Performance Deterioration in Sarcopenia Correlates With Circulating Muscle Tissue-Specific miRNAs
}

\author{
Simona VALÁŠKOVÁ ${ }^{1}$, Andrea GAŽOVÁ ${ }^{2}$, Petra VRBOVÁ ${ }^{3}$, Tomáš KOLLER ${ }^{3}$, \\ Barbara ŠALINGOVA ${ }^{3}$, Adriana ADAMIČKOVÁ ${ }^{3}$, Nikola CHOMANIČOVÁ ${ }^{1}$, \\ Nikoleta HULAJOVÁ ${ }^{4}$, Juraj PAYER ${ }^{3}$, Ján KYSELOVIČ ${ }^{3}$
}

${ }^{1}$ Department of Pharmacology and Toxicology, Faculty of Pharmacy, Comenius University Bratislava, Bratislava, Slovakia, ${ }^{2}$ Institute of Pharmacology and Clinical Pharmacology, Faculty of Medicine, Comenius University Bratislava, Slovakia, ${ }^{3} 5^{\text {th }}$ Department of Internal Medicine, Faculty of Medicine, Comenius University, University Hospital Bratislava, Slovakia, ${ }^{4} 4^{\text {th }}$ Department of Internal Medicine, Faculty of Medicine, Comenius University, University Hospital Bratislava, Slovakia

Received March 17, 2021

Accepted August 27, 2021

\begin{abstract}
Summary
Sarcopenia is defined as an age-associated loss of skeletal muscle function and muscle mass and is common in older adults. Sarcopenia as a disease is currently of interest not only to orthopedists and surgeons but also to internists, endocrinologists, rheumatologists, cardiologists, diabetologists, gynaecologists, geriatricians and paediatricians. In cooperation with the $5^{\text {th }}$ Internal Medicine Clinic, we, as a unit of clinical research, aimed to describe a sarcopenic specific miRNA expression profile for disease diagnostics and classification of the severity of muscle performance deterioration. This study included a total of 80 patients (age $55-86$ years) hospitalized at the V. Internal medicine clinic of LFUK and UNB with different severity of muscle performance deterioration. The study participants were evaluated and classified according to short physical performance battery score (SPPB). In this study, we investigated the role of circulating miRNAs in sarcopenia in the elderly. We hypothesized that sarcopenia effects the expression of muscle tissue-specific miRNAs (MyomiRNAs), which could be potentially reflected in the blood plasma miRNA expression profile. The expression of specific circulating miRNAs in patients with different muscle performances was analyzed. Patients' blood plasma was evaluated for the expression of myomiRNAs: miRNA-29a, miRNA-29b, miRNA-1, miRNA-133a, miRNA-133b, miRNA-206, miRNA-208b and miRNA-499, and the data were correlated with diagnostic indicators of the disease. We showed a specific sarcopenia miRNA profile that could be considered a possible
\end{abstract}

biomarker for the disease. Patients with low muscle performance showed increased miRNA-1, miRNA-29a and miRNA-29b expression and decreased for the miRNA-206, miRNA-133a, miRNA-133b, miRNA-208b and miRNA-499 expression. We show that the severity of muscle performance deterioration in sarcopenia correlates with specific miRNA expression. We also propose the profile of miRNAs expression in blood plasma as a specific biomarker for sarcopenia diagnostics. Future clinical studies will be necessary to eventually naturally have to elucidate the underlined molecular mechanism responsible for specific miRNAs expression in sarcopenia pathology and progression of the disease.

\section{Key words}

Sarcopenia • MiRNAs • Biomarker

\section{Corresponding author}

Andrea Gažová, Institute of Pharmacology and Clinical Pharmacology, Faculty of Medicine, Comenius University Bratislava, Slovak Republic. E-mail: andrea.gazova@fmed.uniba.sk

\section{Introduction}

Sarcopenia was recognized in 2016 as a disease by the World Health Organization (WHO)'s International Statistical Classification of Diseases and Related Health

PHYSIOLOGICAL RESEARCH • ISSN 1802-9973 (online) - an open access article under the CC BY-NC-ND 4.0 license (C) 2021 Institute of Physiology of the Czech Academy of Sciences, Prague, Czech Republic 
Problems (ICD), however the first steps of diagnosis date to the 1980s. In 1989, Rosenberg first proposed the term "sarcopenia" (from the Greek "sarx" for flesh and "penia" for loss) to define a typical age-associated decrease in muscle mass. Today sarcopenia is defined as an age-associated loss of skeletal muscle function and muscle mass and is common in older adults (Dent et al. 2018, Greco et al. 2019). The overall prevalence of sarcopenia is estimated at approximately $6-22 \%$ in adults aged 65 years and increases with age. It is already clear that with such an aging population, the incidence of this disease is increasing (Ethgen et al. 2017). Sarcopenia is associated with increased adverse outcomes including falls, nuctional decline, frailty, and mortality (Björkman et al. 2019). The recognition of sarcopenia as a disease has led to significant advances in research, screening and diagnosis. Sarcopenia is diagnosed based on muscle mass, muscle strength and physical performance assessments. Even though no sarcopenia treatment is currently available, early diagnosis is essential as it reduces the risk of falls, fractures, functional decline, hospitalization and mortality associated with this condition. Management of sarcopenia involves a variety of approaches (Dionyssiotis 2019). Sarcopenia also reduces the amount of metabolically active tissue, which means that it increases metabolic diseases, including cardiovascular diseases, diabetes, hypertension and hyperlipidemia, and other diseases in the elderly. This will bring a heavy economic burden to the medical service system (Biolo et al. 2014).

\section{Sarcopenia in chronic illness}

Sarcopenia is classified as primary sarcopenia when the onset is related to the aging process and secondary, which is related to comorbidities, malnutrition, or immobility. Sarcopenia comorbidities include organ failure (e.g. heart, kidneys, lungs, brain and liver), inflammatory diseases, cancer, and endocrine diseases. Malnutrition due to gastrointestinal disorders, anorexia in cases of polypharmacy, and inadequate intake due to psychosocial disorders can also result in secondary sarcopenia. Finally, inactivity as a result of postoperative deconditioning or prolonged rehabilitation can also result in activity-related secondary sarcopenia (Saggini et al. 2017).

\section{Sarcopenia and microRNA}

MicroRNAs (miRNA) are promising RNA molecules for targeted diagnosis and treatment in the $21^{\text {st }}$ century. This group of RNAs plays a key role in a wide range of physiological and pathological processes. MiRNAs are non-coding regulatory RNA molecules of 20-30 nucleotides in length that regulate gene expression at the post-transcriptional level (Brown a GoljanekWhysall 2015). Growing amount evidence of suggests that circulating miRNAs present in serum or plasma are remarkably stable, easily detectable and can indicate a variety of health changes. It is believed miRNAs presence in serum or plasma can be used as potential biomarkers for the diagnosis and treatment of various diseases. A growing amount of evidence supports circulating miRNAs as a potential therapeutic biomarker for sarcopenia as the expression of specific circulating miRNAs is associated with muscle loss in the elderly (Siracusa et al. 2018).

In this study, we investigated the role of circulating miRNAs in sarcopenia in the elderly. We analysed the expression of specific circulating miRNAs in patients with different muscle performances. We hypothesized that sarcopenia effects the expression of muscle tissue-specific miRNAs (MyomiRNAs), which could be potentially reflected in the blood plasma miRNA expression profile. We analysed the expression of myomiRNAs: miRNA-29a, miRNA-29b, miRNA-1, miRNA-133a, miRNA-133b, miRNA-206, miRNA-208b and miRNA-499 in patient's blood plasma. We correlated these expression levels with diagnostic indicators of sarcopenia and we showed a specific miRNA sarcopenia profile that could be a possible biomarker.

\section{Materials and methods}

The study has been following the proceedings of the Declaration of Helsinki and further amendments. The study protocol and the informed consent form have been approved by the ethics committee of the University Hospital in Bratislava. Study participants signed informed consent before any procedure and agreed with anonymized storage and publication of data.

\section{Participants}

This study included a total of 80 patients (age 55-86 years) hospitalized at the 5th Internal medicine clinic of LFUK and UNB with different severity of muscle performance deterioration. The study participants were evaluated and classified according to short physical performance battery score (SPPB). The short physical performance battery score is the sum of points from three tests: 1 . Chair-stands (s), the time necessary for 5 chair stands without the help of hands, 2. Balance test (s), time 
the patient maintains the balance having feet together, at the semi-tandem and tandem position for maximum 10 seconds in each, 3. Gait Speed, by measuring the time necessary to walk 5 meters $(\mathrm{m} / \mathrm{s})$. Participants were divided into 3 groups according to the SPPB score, evaluated at the sum of individual tests ranging from 0 (not possible) to 4 (best performance). The maximum score is 12 (Table 1). The initial clinical parameters of the patients in the individual groups are shown in Table 2.
Table 1. Division of patients into groups according to the SPPB score.

\begin{tabular}{lcc}
\hline Group & SPPB score & Muscle performance \\
\hline Group 1 & SPPB $\leq 6$ & low muscle performance \\
Group 2 & SPPB 7-9 & moderate muscle performance \\
Group 3 & SPPB $>9$ & high muscle performance \\
\hline
\end{tabular}

Table 2. Gender, age and BMI distribution of the 3 studied sarcopenia patient groups. The data is shown at mean \pm SD.

\begin{tabular}{|c|c|c|}
\hline Clinical parameters & male & female \\
\hline \multicolumn{3}{|c|}{ I. Sarcopenia group $1(n=31)$ SPPB $\leq 6$} \\
\hline Genger & 18 & 13 \\
\hline Age (years) & $70.61 \pm 8.51$ & $75.46 \pm 7.68$ \\
\hline$B M I\left(\mathrm{~kg} / \mathrm{m}^{2}\right)$ & $30.16 \pm 7.97$ & $27.9 \pm 3.89$ \\
\hline \multicolumn{3}{|c|}{ II. Sarcopenia group $2(n=17)$ SPPB 7-9 } \\
\hline Genger & 6 & 11 \\
\hline Age (years) & $64.33 \pm 10.89$ & $69.64 \pm 6.84$ \\
\hline$B M I\left(\mathrm{~kg} / \mathrm{m}^{2}\right)$ & $26.75 \pm 2.57$ & $30.28 \pm 7.32$ \\
\hline \multicolumn{3}{|c|}{ III. Sarcopenia group $3(n=32)$ SPPB $>9$} \\
\hline Genger & 16 & 16 \\
\hline Age (years) & $64.81 \pm 6.44$ & $66.56 \pm 6.27$ \\
\hline$B M I\left(\mathrm{~kg} / \mathrm{m}^{2}\right)$ & $27.4 \pm 3.6$ & $27.9 \pm 3.9$ \\
\hline
\end{tabular}

\section{Plasma sampling}

Venous blood was collected into silicone-coated serum tubes with enhanced silica-containing clot activator $1 / 2 \mathrm{~h}$ after exercise and measurement of anthropometric parameters. At $4{ }^{\circ} \mathrm{C}$, blood samples were centrifuged at 3,500 rpm. Within 10 minutes plasma and erythrocytes were separated. Plasma was immediately aliquoted and frozen at $-80^{\circ} \mathrm{C}$ until further analyses.

\section{MiRNA analysis}

RNA isolation

Before isolation, the plasma was dissolved at room temperature. For stability, repeated freezing and thawing cycles of serum samples were minimized. Isolation was performed on the miRNeasy Serum / Plasma Advanced Kit. Control ce-miRNA-39 (miRNeasy serum/plasma Spike-In control) was added to the process. The process resulted in total RNA eluate from $200 \mu$ of the patient's blood plasma.

\section{Reverse RNA transcription}

Commercially available miRCURY LNA RT Kit (Qiagen) was used for reverse transcription according to the manufacturer's instructions. The resulting cDNA was further analyzed by qPCR.

\section{qPCR method}

The target sequence was analyzed by qPCR miRCURY LNA SYBR ${ }^{\circ}$ Green PCR Kits (Qiagen). The PCR reaction was performed in a StepOnePlus Real-Time PCR System (Life Technologies, USA). Table 3 lists the PCR primers used in this study.

\section{Statistical analysis}

Statistical evaluation was performed by Graphpad using one way ANOVA test and Chi test. Significant changes were considered to be those at which $\mathrm{p}<0.05$. 
Table 3. List of microRNA primers (Qiagen $\mathrm{GmbH}$, Germany)

\begin{tabular}{lccc}
\hline microRNA & LNA PCR primer ID & microRNA & LNA PCR primer ID \\
\hline$c e l-m i R-39-3 p$ & YP00203952 & hsa-miR-133a-3p & YP00204788 \\
$h s a-m i R-1-3 p$ & YP00204344 & hsa-miR-133b & YP00206058 \\
$h s a-m i R-206$ & YP00206073 & hsa-miR-208b-3p & YP00204636 \\
$h s a-m i R-29 a-3 p$ & YP00204698 & hsa-miR-499-5p & YP00205935 \\
$h s a-m i R-29 b-3 p$ & YP00204679 & & \\
\hline
\end{tabular}

Table 4. The values of SPPB test for 3 studied sarcopenia patient group. Each participant underwent a measurement: grip strength, balance, walking speed and chair stand test. The data is shown at mean \pm SD.

\begin{tabular}{lcc}
\hline Anthropometric indexes & male & female \\
\hline & I. Sarcopenia group 1 (n=31) SPPB $\leq \mathbf{6}$ & \\
\hline Grip strength & $20.24 \pm 6.51$ & $14.49( \pm 3.40)$ \\
Balance $(s)$ & $22.61( \pm 4.98)$ & $17.61( \pm 6.37)$ \\
Walking speed $(m / s)$ & $0.47( \pm 0.24)$ & $0.45( \pm 0.17$ \\
Chair stand test $(5 x)$ & $22.11( \pm 8.07)$ & $19.74( \pm 7.46)$ \\
\hline & II. Sarcopenia group 2 (n=17) SPPB 7-9 & \\
\hline Grip strength & $28.61( \pm 3.89)$ & $16.76( \pm 3.26)$ \\
Balance $(s)$ & $26.5( \pm 3.51)$ & $26.36( \pm 3.75)$ \\
Walking speed $(m / s)$ & $0.83( \pm 0.33)$ & $0.75( \pm 0.24)$ \\
Chair stand test $(5 x)$ & $15.25( \pm 5.21)$ & $14.35( \pm 1.91)$ \\
\hline & III. Sarcopenia group 3 (n=32) SPPB $>\mathbf{9}$ & \\
\hline Grip strength & $31.52( \pm 9.80)$ & $19.08( \pm 5.85)$ \\
Balance $(s)$ & $29.44( \pm 2.25)$ & $29.44( \pm 1.79)$ \\
Walking speed $(m / s)$ & $1.03( \pm 0.23)$ & $1.00( \pm 0.29)$ \\
Chair stand test $(5 x)$ & $10.75( \pm 1.87)$ & $11.82( \pm 2.43)$ \\
\hline
\end{tabular}

\section{Results}

In this study, we intended to determine whether the severity of muscle performance deterioration in sarcopenia patients correlates with specific blood plasma miRNA expression profiles. The clinical characteristics for all subjects are shown in Table 1. The patients were divided into 3 groups based on SPPB score (muscle performance deterioration) determined by anthropologic parameters including grip strength, balance, walking speed and chair stand test (Table 4). Biochemical parameters of the clinical study participants were included in the analysis however we did not identify any correlation with the severity of the SPPB score (Table 5). From all the studied clinical parameters participants age alone correlated with the SPPB score. Group 1 showed the highest age (70.61 \pm 8.5 male, $75.46 \pm 7.68$ female) .

In each group, we analyzed the relative expression of miRNA-1, miRNA-29a, miRNA-29b, miRNA-133a, miRNA-133b, miRNA-206, miRNA-208b and miRNA-499 standardized to ce-miRNA-39. The results are shown in Fig. 1.

We showed increased miRNA-1 expression in group 1, patients with the lowest muscle performance, however, the difference was not significant. We observed a significant increase in miRNA-1 expression only between groups 2 and 3. However, we observed a decrease in miRNA-206 expression in the group of patients with the weakest muscle performance (Fig. 1A). The expression of miRNA-133a and miRNA-133b decreased in the group of patients with low muscle performance (Fig. 1B). In the miRNA-29 family, we did not observe any significant changes in either miRNA-29a expression or miRNA-29b (Fig. 1C). The expression of miRNA-208b and miRNA-499 decreased in patients with the lowest muscle output (Fig. 1D). 
Table 5. Biochemical parameters of all the studied sarcopenia patient group. The data is shown at mean \pm SD.

\begin{tabular}{lcc}
\hline Biochemical parameters & male & female \\
\hline \multicolumn{3}{l}{ I. Sarcopenia group $\mathbf{1}(\mathbf{n}=\mathbf{3 1}) \mathbf{S P P B} \leq \mathbf{6}$} \\
\hline Hemoglobin & $118.94( \pm 17.18)$ & \\
Bilirubin & $17.20( \pm 12.85)$ & $120.05( \pm 21.31)$ \\
Urea & $8.64( \pm 5.55)$ & $10.99( \pm 7.21)$ \\
Kreatinin & $110.28( \pm 61.47)$ & $7.80( \pm 5.05)$ \\
CRP & $49.02( \pm 77.98)$ & $125.01( \pm 107.86)$ \\
D-vitamin & $16.23( \pm 9.56)$ & $15.12( \pm 25.40)$ \\
\hline
\end{tabular}

\section{Sarcopenia group $2(n=17)$ SPPB 7-9}

\begin{tabular}{lcc}
\hline Hemoglobin & $109( \pm 21.50)$ & $121.67( \pm 17.17)$ \\
Bilirubin & $47.07( \pm 70.10)$ & $21.03( \pm 23.14)$ \\
Urea & $11.00( \pm 7.51)$ & $10.13( \pm 15.95)$ \\
Kreatinin & $121.93( \pm 68.91)$ & $77.56( \pm 19.81)$ \\
CRP & $17.472( \pm 23.36)$ & $8.61( \pm 10.02)$ \\
D-vitamin & $15.18( \pm 8.31)$ & $17.75( \pm 2.43)$ \\
\hline
\end{tabular}

\begin{tabular}{lcc}
\hline \multicolumn{3}{c}{ III. Sarcopenia group $\mathbf{3}(\mathbf{n}=\mathbf{3 2}) \mathbf{S P P B}>\mathbf{9}$} \\
\hline Hemoglobin & $123.45( \pm 29.20)$ & $116.5( \pm 15.73)$ \\
Bilirubin & $144.06( \pm 466.31)$ & $15.24( \pm 24.03)$ \\
Urea & $5.66( \pm 1.67)$ & $4.70( \pm 2.97)$ \\
Kreatinin & $89.81( \pm 27.57)$ & $76.58( \pm 16.84)$ \\
CRP & $37.57( \pm 67.06)$ & $30.44( \pm 35.17)$ \\
D-vitamin & $19.91( \pm 9.44)$ & $17.65( \pm 9.37)$ \\
\hline
\end{tabular}

\section{Discussion}

Aging of the musculoskeletal system is a common health problem today. Sarcopenia as a disease is currently of interest not only to orthopedists and surgeons but also to internists, endocrinologists, rheumatologists, cardiologists, diabetologists, gynecologists, geriatricians and pediatricians. Many unanswered questions give us room to examine more deeply the issue of this disease in terms of proper diagnosis and treatment, as well as clinical complications. In cooperation with the 5 th Internal Medicine Clinic, we, as a unit of clinical research, aimed to describe a sarcopenic specific miRNA expression profile for disease diagnostics and classification of the severity of muscle performance deterioration. We examined blood plasma patient samples for expression of specific myomiRNA expression. We chose blood plasma analysis as it is generally preferred for diagnostic purposes, while an easily obtainable material in the clinical setting. Compared with muscle biopsy it is a significantly less invasive procedure for the patient.

In this study, we characterized and analyzed plasma samples from hospitalized patients diagnosed with sarcopenia. Multiple studies suggest that impaired expression of myogenic miRNAs may result in the development of various pathological conditions of muscle apparatus. The study participants were divided into 3 groups based on the SPPB score value and in each of them we examined the expression of myomiRNAs as miRNA-1, miRNA-206, miRNA-29a, miRNA-29b, miRNA-133a, miRNA-133b, miRNA-208 and miRNA-499. The analysis showed a specific miRNA expression profile for each of the 3 studied groups. Our data suggest a miRNA expression correlation with muscle performance deterioration in sarcopenia and indicate miRNA role in the disease progression.

MiRNA-133a and miRNA-133b regulate the basic processes of myogenesis, including differentiation, regeneration, and determination of the fate of myoblast satellite cells (Cui et al. 2019, Iannone et al. 2020). Its overexpression has been shown to occur during 
miRNA-1

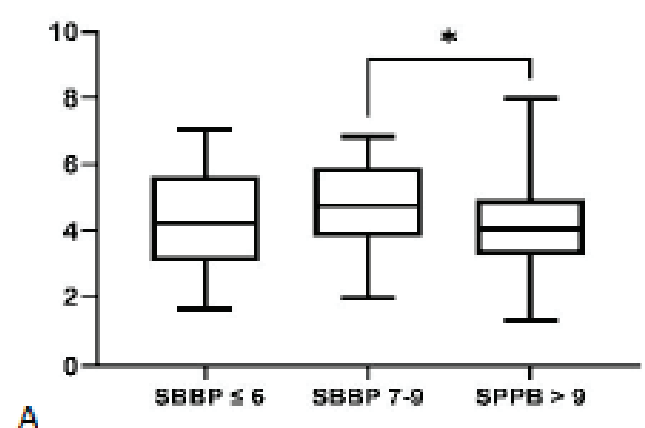

miRNA-133a

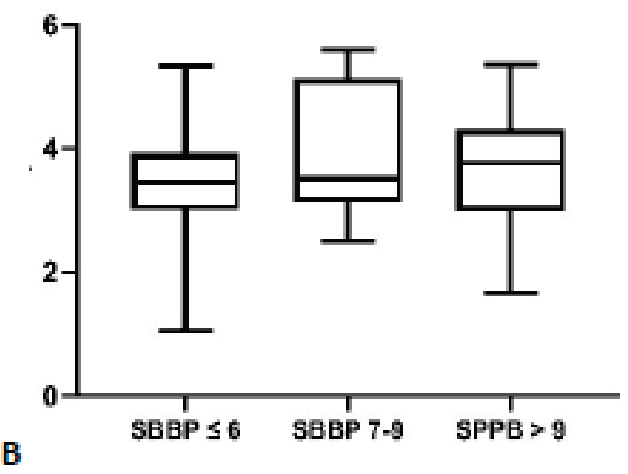

miRNA-29a

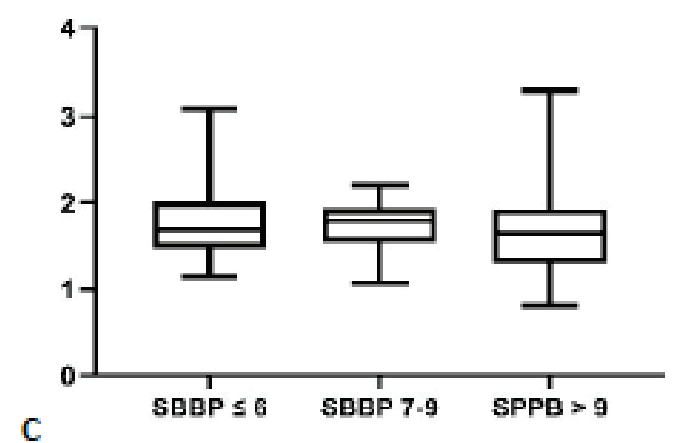

miRNA-499

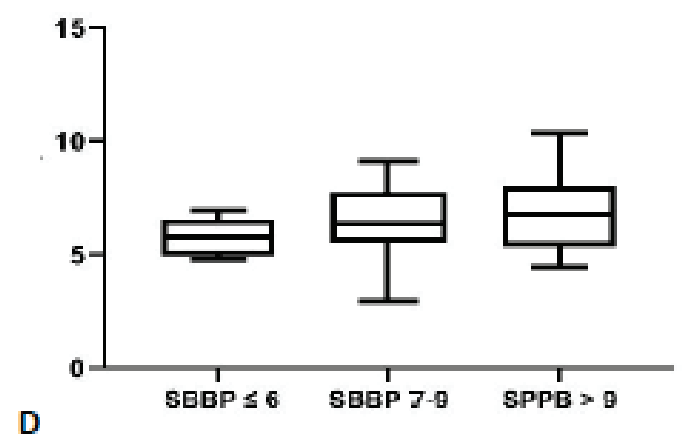

miRNA-206

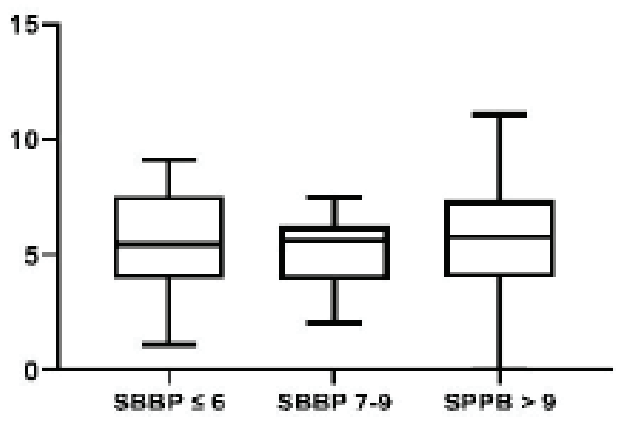

miRNA-133b

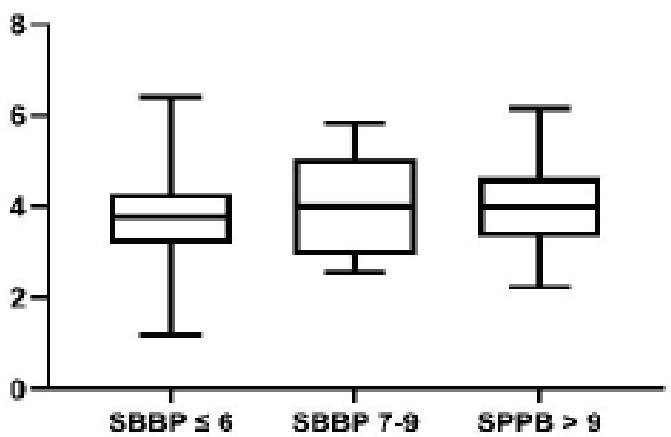

miRNA-29b

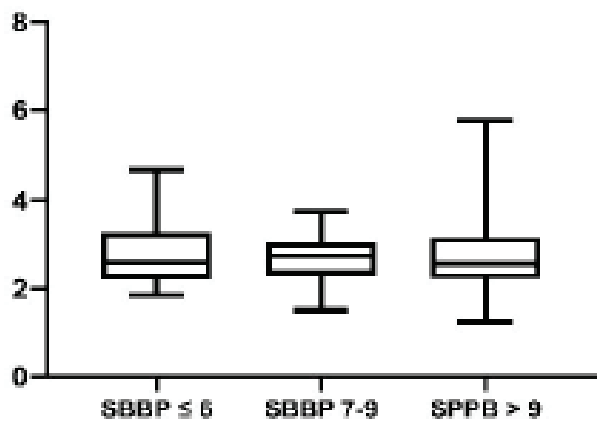

MiRNA-208b

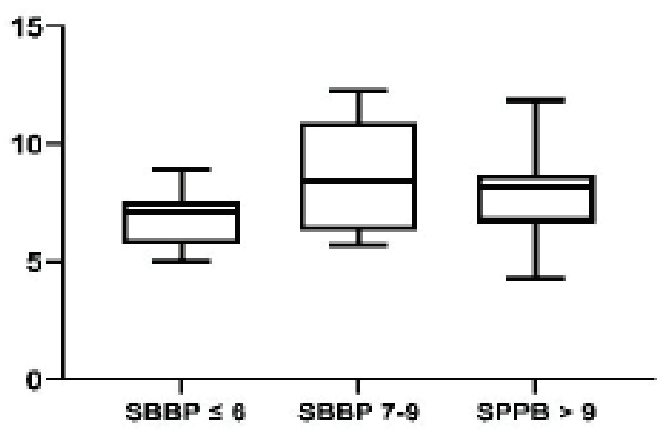

Fig. 1. A) The expression of miRNA-1 and miRNA-206 in individual groups of patients with different muscle performance deterioration standardized to ce-miRNA-39. B) The expression of miRNA-133a and miRNA-133b in individual groups of patients with different muscle performance deterioration standardized to ce-miRNA-39. C) The expression of miRNA-29a and miRNA-29b in individual groups of patients with different muscle performance deterioration standardized to ce-miRNA-39. D) The expression of miRNA-208b and miRNA-499 in individual groups of patients with different muscle performance deterioration standardized to ce-miRNA-39. 
myogenesis (Horak et al. 2016), while its downregulation causes muscle cell weakening, a hallmark of sarcopenia. Sarcopenic muscle also shows a low regenerative capacity and impaired differentiation potential (SousaVictor and Muñoz-Cánoves 2016). This miRNA family play an important role in the modulation of stem cell function, and activity, muscle homeostasis, and are implicated in various neuromuscular diseases. Specifically, Chen et al. showed that loss of miRNA in muscle leads to a reduction in muscle cell number, and results in mild atrophy and impaired regenerative capacity. Only recently, several studies showed that miR-1, miR-206, miR-133a and miRNA-133b could be potential biomarkers in muscle regeneration. Increased expression of the listed myomiRNAs promotes muscle cell differentiation and regeneration (Chen et al. 2006, Kim et al. 2006). Our data support the hypothesis that downregulation of miR-133b (part of the miRNA-133 family) may contribute to a reduction in the myogenic and regenerative capacity of muscle cells in patients with sarcopenia., discussed previously in multiple studies (Yu et al. 2014, Yanai et al. 2020). We also showed the similar expression pattern in miRNA-206.

Van Rooij et al. (2009) studied the expression of muscle-specific miRNAs, specifically miRNA-208a, miRNA-208b and miRNA-499, in multiple research reports. All three myomiRNAs are transcripts of three myosin genes. He discovered that miR-208a is cardiac muscle-specific and is essential for the regulation of miR$208 \mathrm{~b}$, which is also found in cardiac muscle but is highly specific for skeletal muscle as well as miRNA-499. Their expression in skeletal muscle is limited to slow muscle fibers and also plays a key role in muscle fiber displacement and muscle growth promotion (van Rooij et al. 2009). In our study, we observed a decrease in expression of myomiRNA, miRNA-208b and miRNA499 in patients with low muscle performance. This finding suggests an inability to grow muscle mass in patients with sarcopenia, which demonstrates a decrease in SPPB value.

Only a few studies have previously addressed the expression of miRNA-29a and miRNA-29b in people with sarcopenia. The study by $\mathrm{Hu}$ et al. in 2014 indicated an increase in miRNA-29 expression at an older age, providing a potential mechanism for aging-induced sarcopenia (Hu et al. 2014). In our study, however, we did not observe any significant difference in miRNA-29 expression between patient groups.

It should be noted that very few studies have previously studied a relationship between changes in plasma/serum expression and muscle miRNAs in sarcopenia. Only a limited number of studies have considered the miRNA role in disease progression or diagnostics. We show that the severity of muscle performance deterioration in sarcopenia correlates with specific miRNA expression. We also propose the profile of miRNAs expression in blood plasma as a specific biomarker for sarcopenia diagnostics. Future clinical studies will naturally have to elucidate the underlined molecular mechanism responsible for specific miRNAs expression in sarcopenia pathology and the progression of the disease.

\section{Conflict of Interest}

There is no conflict of interest.

\section{References}

BIOLO G, CEDERHOLM T, MUSCARITOLI M: Muscle contractile and metabolic dysfunction is a common feature of sarcopenia of aging and chronic diseases: from sarcopenic obesity to cachexia. Clin Nutr 33: 737-748, 2014. https://doi:10.1016/j.clnu.2014.03.007

BJÖRKMAN M, JYVÄKORPI S.K, STRANDBERG TE, PITKÄLÄ KH, TILVIS RS: Sarcopenia indicators as predictors of functional decline and need for care among older people. J Nutr Health Aging 23: 916-922, 2019. https://doi.org/10.1007/s12603-019-1280-0

BROWN D, GOLJANEK-WHYSALL K: microRNAs: Modulators of the underlying pathophysiology of sarcopenia? Ageing Res Rev 24: 263-273, 2015. https://doi:10.1016/j.arr.2015.08.007

CHEN JF, MANDEL EM, THOMSON JM, WU Q, CALLIS TE, HAMMOND SM, CONLON FL, WANG DZ. The role of microRNA-1 and microRNA-133 in skeletal muscle proliferation and differentiation. Nat Genet 38 : 228-233, 2006. https://doi:10.1038/ng1725

CUI S, LI L, MUBAROKAH SN, MEECH R: Wnt/ $\beta$-catenin signaling induces the myomiRs miR-133b and miR-206 to suppress IPax7 and induce the myogenic differentiation program. J Cell Biochem 120: 12740-12751, 2019. https://doi:10.1002/jcb.28542 
DENT E, MORLEY JE, CRUZ-JENTOFT AJ, ARAI H, KRITCHEVSKY SB, GURALNIK J, BAUER JM, PAHOR M, CLARK BC, CESARI M, RUIZ J, SIEBER CC, AUBERTIN-LEHEUDRE M, WATERS DL, VISVANATHAN R, LANDI F, VILLAREAL DT, FIELDING R, WON CW, THEOU O, MARTIN FC, DONG B, WOO J, FLICKER L, FERRUCCI L, MERCHANT RA, CAO L, CEDERHOLM T, RIBEIRO SML, RODRÍGUEZ-MAÑAS L, ANKER SD, LUNDY J, GUTIÉRREZ ROBLEDO LM, BAUTMANS I, APRAHAMIAN I, SCHOLS JMGA, IZQUIERDO M, VELLAS B: International Clinical Practice Guidelines for Sarcopenia (ICFSR): Screening, Diagnosis and Management. J Nutr Health Aging 22:1148-1161. 2018. https://doi:10.1007/s12603-018-1139-9

DIONYSSIOTIS Y: Sarcopenia in the elderly. Eur Endocrinol 15: 13-14, 2019. https://doi:10.17925/EE.2019.15.1.13

ETHGEN O, BEAUDART C, BUCKINX F, BRUYĖRE O, REGINSTER JY: The future prevalence of sarcopenia in Europe: a claim for public health action. Calcif Tissue Int 100: 229-234, 2017. https://doi:10.1007/s00223-0160220-9

GRECO EA, PIETSCHMANN P, MIGLIACCIO S: Osteoporosis and sarcopenia increase frailty syndrome in the elderly. Front Endocrinol 10: 255, 2019. https://doi:10.3389/fendo.2019.00255

HORAK M, NOVAK J, BIENERTOVA-VASKU J: Muscle-specific microRNAs in skeletal muscle development. Dev Biol 410: 1-13. 2016. https://doi:10.1016/j.ydbio.2015.12.013

HU Z, KLEIN J D, MITCH W E, ZHANG L, MARTINEZ I, WANG XH: MicroRNA-29 induces cellular senescence in aging muscle through multiple signaling pathways. Aging 6:160, 2014. https://doi:10.18632/aging.100643

IANNONE F, MONTESANTO A, CIONE E, CROCCO P, CAROLEO MC, DATO S, ROSE G, PASSARINO G. Expression patterns of muscle-specific miR-133b and miR-206 correlate with nutritional status and sarcopenia. Nutrients 12: 297, 2020. https://doi:10.3390/nu12020297

KIM H K, LEE YS, SIVAPRASAD U, MALHOTRA A, DUTTA A: Muscle-specific microRNA miR-206 promotes muscle differentiation. J Cell Biol 174: 677-687, 2006. https://doi:10.1083/jcb.200603008

SAGGINI R, CARMIGNANO SM, COSENZA L, PALERMO T, BELLOMO R: Sarcopenia in Chronic Illness and Rehabilitative Approaches. Frailty and Sarcopenia - Onset, Development and Clinical Challenges 2017. https://doi:10.5772/intechopen.70223

SIRACUSA J, KOULMANN N, BANZET S. Circulating myomiRs: a new class of biomarkers to monitor skeletal muscle in physiology and medicine. J Cachexia Sarcopenia Muscle 9: 20-27. 2018. https://doi:10.1002/jcsm.12227

SOUSA-VICTOR P, MUÑOZ-CÁNOVES P: Regenerative decline of stem cells in sarcopenia. Mol Aspects Med 50: 109-117, 2016. https://doi:10.1016/j.mam.2016.02.002

VAN ROOIJ E, QUIAT D, JOHNSON BA, SUTHERLAND LB, QI X, RICHARDSON JA, KELM RJ JR, OLSON EN: A family of microRNAs encoded by myosin genes governs myosin expression and muscle performance. Dev Cell 17: 662-673, 2009. https://doi:10.1016/j.devcel.2009.10.013

YANAI K, KANEKO S, ISHII H, AOMATSU A, ITO K, HIRAI K, OOKAWARA S, ISHIBASHI K, MORISHITA Y: MicroRNAs in sarcopenia: a systematic review. Front Med 7: 180, 2020. https://doi:10.3389/fmed.2020.00180

YU H, LU Y, LI Z, WANG Q: microRNA-133: expression, function and therapeutic potential in muscle diseases and cancer. Curr Drug Targets 15: 817-828, 2014. https://doi:10.2174/1389450115666140627104151

YEN PM: Physiological and molecular basis of thyroid hormone action. Physiol Rev 81: 1097-1142, 2001. https://doi:10.1152/physrev.2001.81.3.1097 